\title{
Dynamic Functional Unit Assignment for Low Power
}

\author{
Steve Haga \\ Department of Electrical \& Computer Engineering \\ University of Maryland \\ College Park, MD 20742, U.S.A \\ Natasha Reeves \\ Department of Electrical \& Computer Engineering \\ University of Maryland \\ College Park, MD 20742, U.S.A \\ Rajeev Barua \\ Department of Electrical \& Computer Engineering \\ University of Maryland \\ College Park, MD 20742, U.S.A \\ Diana Marculescu \\ Department of Electrical \& Computer Engineering \\ Carnegie Mellon University \\ 5000 Forbes Avenue \\ Pittsburg, PA 15213, U.S.A
}

\begin{abstract}
A hardware method for functional unit assignment is presented, based on the principle that a functional unit's power consumption is approximated by the switching activity of its inputs. Since computing the Hamming distance of the inputs in hardware is expensive, only a portion of the inputs are examined. Integers often have many identical top bits, due to sign extension, and floating points often have many zeros in the least significant digits, due to the casting of integer values into floating point, and other reasons. The accuracy of these approximations is studied and the results are used to develop a simple, but effective, hardware scheme.
\end{abstract}

\section{Keywords}

Low power, Bit patterns, Functional unit assignment, dynamic power, hamming distance. 


\section{Introduction}

Power consumption has become a critical issue in microprocessor design, due to increasing computer complexity and clock speeds. Many techniques have been explored to reduce the power consumption of processors. Low power techniques allow increased clock speeds and battery life.

Power consumption can be reduced at multiple levels - at the architecture level, at the compiler and OS levels, and at the VLSI levels. This paper focuses on a set of techniques to reduce the power consumption of functional units in superscalar processors primarily at the architecture level, but we also consider the impact of a related compiler transformation on power consumption.

There are two sources of power consumption in VLSI chips - static and dynamic power. Static power is defined as the power that is dissipated even without any switching activity in the circuit. Dynamic power is the additional power dissipated when signals in the chip change from a 0 to 1, or vice-versa. In CMOS logic, leakage power is the only source of static power consumption. Static power consumption has been traditionally ignored since it has been negligible, however it is becoming more significant with the downward-scaling of transistor dimensions. Therefore opportunities for significant power reduction are available in both static and dynamic power. This paper focuses on techniques to reduce dynamic power only; static power is not considered further.

The underlying intuition of the schemes in this paper is that by looking at just a few bits of the operand data words, which we call the information bits, we can determine which bit value is likely to be more common in the remaining bits. For integer values, we observe that the sign bit is equal to the leading bits on the left of the number resulting from its sign-extension. Thus a good information bit is the sign bit itself, since the sign bit is more likely in the remaining bits, and thus is a predictor of the frequency of the remaining bits. For example, the decimal number 20 is 0x14 in binary, represented in hexadecimal. In a 32-bit data word, decimal 20 is sign-extended to 
0x00000014, and thus 27 of its leading bits are zero. Similarly, decimal -20 is 0xFFFFFFEC, and thus 27 of its leading bits are one. In both these cases the sign bit is more common in the remaining bits. That this trend is true on average is borne out by our results in section 4.2.

For floating point values, the intuition we use is that many floating point mantissa values have a lot of trailing zeros, for reasons detailed in section 4.2. We have found that the OR of the least significant four bits of the mantissa is a good information bit for prediction: when this information bit is zero, it is likely that a large number of trailing zeros exist. We demonstrate that this prediction is good enough to be useful in section 4.2.

We propose methods for both integer and floating point data to benefit from this intuition in terms of power savings. For integer values we use multiple identical functional units that are present in superscalars, but instead of treating them as interchangeable, we always assign instructions to them based on the sign bits of instruction operands. For example, for the two input operands for an instruction, there are four possible combinations of sign bits: 00, 01, 10 and 11 . If four functional units are present, then we can pre-assign one case to each unit. The advantage of doing so is that since successive operations to the same functional unit share a lot of bits, dynamic power dissipation is minimized. It is not necessary that four functional units be present; power savings can be achieved with two or more functional units. For floating point adder/subtracter units, an idea similar to that for integer units can be used. The actual scheme is somewhat different from this simple idea and is described in sections 4 and 5 .

With this technique, we succeed in reducing the power of integer ALU operations by $17 \%$ and the power of floating point operations by $18 \%$. In [4] it was found that around $22 \%$ of the processor's power is consumed in the execution units. Thus, the decrease in total chip power is roughly $4 \%$. While this overall gain is modest, two points are to be noted. First, one way to reduce 
overall power is to combine various techniques such as ours, each targeting a different critical area of the chip. It is unlikely that a single 'silver bullet' method for power savings will reduce power across all areas of the chip and across all layers of abstraction. Thus low power designs in the future will incorporate a large number of individual technologies for power reduction, one of which could be ours. Second, reducing the execution units' power consumption by $17 \%$ to $18 \%$ is desirable, independent of the overall effect, because the execution core is one of the hot-spots of power density within the processor, and is at a risk of burn out.

We also present an independent compiler optimization called swapping that further improves the gain for integer ALU operations to 26\%. The intuition behind swapping is that sometimes the number of bit value changes can be reduced on average by switching the order of commutative operations. Operand swapping in both hardware and software is investigated.

Our approach has also been extended to multiplier FUs; these units present a different set of problems. Even modern superscalars tend to only have one integer and one floating point multiplier. Hence reducing power by steering to one of multiple functional units is not feasible for multiplication. However the following observation allows for savings using a different approach: in the case of the Booth multiplier, the power is known to depend not only on the switching activity of the operands, but also on the number of 1's in the second operand [15]. Thus power savings are possible by swapping the operands to ensure that the second operand is the one with fewer ones in it. Section 4.4 discusses the swapping optimization. Finally, power aware routing is not beneficial for FUs that are not duplicated and not commutative, such as the divider FU. 


\section{Energy modeling in functional units}

This section presents a simple model for the dynamic power dissipation in functional units. Static power is not targeted and thus is not modelled in this paper. To begin, it is known [18] that the most important source of power dissipation in a module is the dynamic charging and discharging of its gates, called the switched capacitance. This switched capacitance is dependent upon the module's input values [17]. It has been further shown [16, 6] that the Hamming distance of consecutive inputs, defined as the number of bit positions that differ between them, provides a suitable measure of power consumption. Thus the more the number of bits that change value between successive computations, the greater the power consumption. In $[16,6]$, power is modeled as: Power $\approx \frac{1}{2} V_{d d}^{2} f \sum_{k} C_{k} s w_{k} \approx \frac{1}{2} V_{d d}^{2} f C_{\text {module }} h_{\text {input }}$, where $V_{d d}=$ voltage, $f=$ the clock frequency, $C_{k}=$ the capacitance of output gate $k, s w_{k}=$ the average number of transitions for output gate $k$ (called switching activity), $C_{\text {module }}=$ the total capacitance of the module, and $h_{\text {input }}=$ the Hamming distance of the current inputs to the previous ones.

Since power consumption is approximately linearly proportional to $h_{\text {input }}$, it is desirable to

minimize $h_{\text {input }}$. Such a minimization is possible because modern processors contain multiple integer arithmetic logic units, or IALUs, and multiple floating point arithmetic units, or FPAUs. IALU and FPAU are types of functional units, or FUs. Current superscalars assign operations to the FUs of a given type without considering power; a better assignment can reduce power, however, as illustrated by the example in Figure 1, which shows the input operands to three identical FUs, in two successive cycles. The alternate routing consumes less power because it reduces the Hamming distance between cycles 1 and 2 . Figure 1 assumes that the router has the ability to not only assign an operation to any FU, but also to swap the operands, when beneficial and legal. For instance, it is legal to swap the operands of an add operation, but not those of a subtract. (A subtract's operands 
can be swapped if they are first inverted, but the cost outweighs the benefit). Because superscalars allow out-of-order execution, a good assignment strategy should be dynamic. The case is less clear for VLIW processors, yet some of our proposed techniques are also applicable to VLIWs.

\section{Related work}

A variety of hardware techniques have been proposed for power reduction, however these methods have not considered functional unit assignment. Existing hardware techniques are generally independent of our method. For example, implementing a clock-slowing mechanism along with our method will not impact the additional power reduction that our approach achieves.

Work has also been done to reduce the power of an application through compiler techniques [15] such as improved instruction scheduling, memory bank assignment, instruction packing and operand swapping. The difficulty of compile time methods is that the power consumed by an operation is highly data dependent [13], and the data behavior dynamically changes as the program executes. A more detailed discussion is found in section 4.4. Further, we study the interaction of compiler swapping with our method in the results in section 6 , and show that the benefits from compiler swapping are mostly orthogonal to, and thus additive with, our hardware methods.

The method in [20] aims to reduce the power consumption of instruction fetch in VLIW

processors. Power reduction is achieved by re-arranging instructions in VLIW long words for reducing bit value changes between long words. Their method relies upon knowing the instructions in the compiler, but since data values are not known at compile-time, a similar idea cannot be applied to reducing power in functional units.

The problem of statically assigning a given set of operations to specific functional units has also been addressed in the context of high-level synthesis for low power [6]. In this scenario, the goal 
is to map operations in a hardware-description language specification to functional units in custom logic at synthesis-time. In [6], the authors present an optimal algorithm for assigning operations to existing resources in the case of data flow graphs (DFGs) without loops. More recently, [14] shows that the case of DFGs with loops is NP-complete, and proposes an approximate algorithm. A key difference with our case is that in high-level synthesis, the assignment is done at synthesis time, so data values of operands are not available, but profile-data may be. In our case of a general-purpose superscalar processor, data values are available, so opportunities for dynamic schemes like ours are available. Functional unit mapping technologies for high-level synthesis do not apply to generalpurpose superscalars since in a superscalar, multiple identical functional units of a single type are not visible from the software, so runtime schemes are essential.

A runtime scheme for reducing power consumption in custom logic has been proposed [8] that reduces power in the case that operand bit-widths are often less that the maximum data width. It divides the functional units into two portions - the less vs. more significant portions, and power reduction is seen if the data width fits within the less significant portion, in which case the more significant portion can be turned off, and its result bits computed by a simple sign-extension circuit instead. The work in [7] uses a similar intuition, but has different details. The major difference of their methods with our method is that they targets a different source of power reduction - low bit width within one computation - rather than similarities in bits across different computations as we do. Thus, the methods are complementary: one can imagine a hybrid scheme where our method is used, but each functional unit is one of theirs, and improvements gained will be additive. Unfortunately, their method may be less effective in general-purpose processors like we target, since the relative sizes of the less and more significant portions of the functional unit are application-specific; such customization is possible only in custom logic. 
The method in [19] reduces power by using heterogeneous functional units, some optimized for performance and some for power. The former are used for critical instructions, and non-critical instructions use the latter. Critical instructions, predicted by a hardware predictor, are those which are believed to be on the critical path of execution, and thus are important to optimize for runtime. Our methods are complementary: one can imagine a hybrid scheme where multiple functional units are available as in our scheme, but two versions of each unit are available, one optimized for performance and the other for power. Such a hybrid combines the benefits of both schemes.

Several hardware techniques have also been explored, which target reducing power in a variety of ways that are unrelated to our method. One technique, [3], is similar to our approach, in that it identifies narrow-width operands, but is not similar in the terms of how the identified operands are optimized. In [3], this information is used to pack several operands into one register. The work in [10] reduces static leakage power in functional units, rather than dynamic power like we do.

\section{Functional unit assignment for low power}

In this section we present an approach for assigning operations to specific FUs that reduces the total switched capacitance of the execution stage. As shown in Figure 1, we can choose a power-optimal assignment by minimizing the switching activity on the inputs.

Our main assumption is that whenever an FU is not used, it has little or no dynamic power consumption. This is usually achievable via power management techniques $[21,1,2]$ which use transparent latches to keep the primary input values unchanged whenever the unit is not used. We will also use this hardware feature for our purposes, and thus, we assume that it is already in place for each of the FUs. To reduce the effect of leakage current when an FU is idle, techniques such as those in [12] may also be used, if needed. 
Some nomenclature is helpful in the following discussion: $\mathbf{M}_{j}:$ the $j_{t h}$ module of the given FU type.

$\mathbf{I}_{j}:$ the $j_{t h}$ instruction to execute this cycle on this FU type.

Num(M), Num(I): \# of modules of type M, or \# of instructions of the given type. The maximum value for $\operatorname{Num}(\mathrm{I})$ is $\mathrm{Num}(\mathrm{M})$, indicating full usage of that FU type.

$\operatorname{OP1}\left(\mathbf{M}_{j}\right), \mathbf{O P 2}\left(\mathbf{M}_{j}\right)$ : the first and second operands of the previous operation performed on this module.

$\mathbf{O P 1}\left(\mathbf{I}_{j}\right), \mathbf{O P 2}\left(\mathbf{I}_{j}\right)$ : the first and second operands of the given instruction.

Commutative $\left(\mathbf{I}_{j}\right)$ : indicates if $\mathbf{I}_{j}$ is commutative.

$\operatorname{Ham}(\mathbf{X}, \mathbf{Y})$ : the Hamming distance between the numbers $\mathrm{X}$ and $\mathrm{Y}$. For floating point values, only the mantissa portions of the numbers are considered.

\subsection{The optimal assignment}

Although its implementation cost is prohibitive, we first consider the optimal solution, so as to provide intuition. On a given cycle, the best assignment is the one which minimizes the total Hamming distance for all inputs. To find this, we compute the Hamming distance of each input operand to all previous input operands. The algorithm to compute these costs is shown in Figure 2. Once these individual costs have been computed, we examine all possible assignments and choose the one which has the smallest total cost (defined as the sum of its individual costs).

This algorithm is not practical. Since the routing logic lies on the critical path of the execution stage, such lengthy computations are sure to increase the cycle time of the machine. Moreover, the power consumed in computing so many different Hamming distances is likely to exceed the power 
savings from inside the modules.

\subsection{Approximate Hamming distance computation}

To reduce the overhead of the above strategy, full Hamming distance computations must be avoided. We achieve this by exploiting certain properties of numerical data to represent an operand by a single information bit.

Integer bit patterns For integer values, we observe that the sign bit is equal to the leading bits on the left of the number resulting from its sign-extension. Thus a good information bit is the sign bit itself, since the sign bit is more likely in the remaining bits, and thus is an approximate predictor of the frequency of the remaining bits. For example, the decimal number 20 is $0 x 14$ in binary, represented in hexadecimal. In a 32-bit data word, decimal 20 is sign-extended to 0x00000014, and thus 27 of its leading bits are zero. Similarly, decimal -20 is 0xFFFFFFEC, and thus 27 of its leading bits are one. In both these cases the sign bit is more common in the remaining bits. This effect is more pronounced in small-magnitude integers, but is present in most integers.

Table 1 demonstrates that the sign bit is a good information bit on average. This table records the operand bit patterns of our benchmarks. (Section 6 describes the benchmarks and how they are run.) By combining the data from the table using probability methods, we find that on average, when the top bit is 0 , so are $91.2 \%$ of the bits, and when this bit is 1 , so are $63.7 \%$ of the bits.

Floating point bit patterns Floating point numbers are represented in scientific notation computers by a mantissa (representing the significant digits with a number between 0 and 1) and an exponent (representing the appropriate power of two). For example, the number 7.0 is represented in binary scientific notation as $111 \times 2^{0}=0.111 \times 2^{3}$. Here the mantissa is 111 and the exponent is $3=11$ in binary. Since the first bit of the mantissa is always 1 in normalized floating point, it is 
usually not stored, and thus the mantissa is also stored as 11 .

Although not as common as in integers, several floating point operands also contain only a few bits of precision in their mantissas, for three main reasons: (1) the casting of integer values into floating point representation, such as happens when incrementing a floating point variable, (2) the casting of single precision numbers into double precision numbers by the hardware because there are no separate, single-precision registers and FUs, and (3) the common use of round numbers in many programs. For example, we saw in the previous paragraph that the number 7.0 has a mantissa of 11. When stored in the 64-bit IEEE standard format, which has a 52 bit mantissa, storing 11 in the mantissa results in 50 trailing zeros.

The presence of a large number of trailing zeros is identified by computing the OR of the leastsignificant four bits of the mantissa as the information bit. The intuition is that if all four of the bottom bits are zero, it is quite likely that a large number of the trailing bits are zero. This is because if full precision numbers have a probability of $1 / 2$ that any one bit is zero, then using four bits misidentifies only $\frac{1}{16}$ of the full-precision numbers. We do not wish to use more than four bits, so as to maintain a fast circuit. Although we refer to an OR gate because it is more intuitive, a NOR gate is equally effective and slightly faster.

Table 1 evaluates the power of the information bit in predicting a large number of trailing zeros for floating point data. From Table 1 we can derive that $42.4 \%$ of floating point operands have zeroes in their bottom 4 bits, implying that $3.8 \%$ of these are full precision numbers that happen to have four zeroes $((100-42.4) \div 15)$ and that the remaining $38.6 \%$ do indeed have trailing zeroes $(42.4-3.8)$. This table also shows that, on average, when the bottom four bits are zero, $86.5 \%$ of the bits are zero. 


\subsection{A lightweight approach for operand steering}

Next we describe how the intuition about the information bit can be used in a method to reduce power consumption. The power reduction method that follows is the same for both integer ALUs and for floating point adder/subtractor units, and applies equally to both, but with different definitions of the information bit, defined earlier.

In section 4.2, the Hamming distance computations of Figure 2 are reduced to just the Hamming distances of the information bits of the operands; this section examines avoiding the computation of Figure 2 entirely. This is achieved by predetermining the FU assignment for any particular set of instruction operands - without comparison to previous values. Some additional definitions are useful:

bit(operand): the information bit of the operand.

$\operatorname{case}\left(\mathbf{I}_{j}\right)$ : the concatenation of $\operatorname{bit}\left(O P 1\left(I_{j}\right)\right)$ with $\operatorname{bit}\left(O P 2\left(I_{j}\right)\right)$. case classifies the instructions into four possible tuples $(00,01,10,11)$.

least: the case with the lowest frequency, as found by examining Table 1 for all four cases, where the commutative and non-commutative rows are combined into one value.

vector: the concatenation of $\left(\operatorname{case}\left(I_{1}\right), \operatorname{case}\left(I_{2}\right), \ldots, \operatorname{case}\left(I_{N u m(I)}\right)\right)$. The size of vector is $2 \times \operatorname{Num}(\mathrm{M})$. If $\mathrm{Num}(\mathrm{I})<\operatorname{Num}(\mathrm{M})$, the remaining bit pairs of vector are set to the least case.

The insight behind an approach of just using present inputs without considering the previous ones is that, by distributing the various instruction cases across the available modules, subsequent instructions to that module are likely to belong to the same case, without needing to check the previous values. For example, if we consider a machine where $\operatorname{Num}(M)=4$, and with an equal probability of each of the four cases $(00,01,10$, and 11), it is logical to assign each of the cases to 
a separate module. In cycles when no more than one instruction of each case is present, this strategy will place them perfectly, even without checking previous values. When multiple instructions of the same case are present, however, the assignment will be non-ideal.

The algorithm is implemented as follows. During each cycle, vector is used as the input address to a look up table, or LUT. The output of that table encodes the assignment of the operations to modules of the given FU. Therefore, the LUT contains the assignment strategy. Although the algorithm is conceptually visualized as using an LUT, the actually implemented circuit may use combinational logic, a ROM, or another method.

The contents of the LUT are determined as follows. The information from Table $1-$ along with new information from Table 2, which lists the probabilities of multiple instructions executing on the given FU type - is used to compute the probabilities of different input patterns. For instance, in the IALU, case 00 is by far the most common $(40.11 \%+29.38 \%=69.49 \%)$, so we assign three of the modules as being likely to contain case 00 , and we use the fourth module for all three other cases (our test machine has 4 modules). For floating point, case 11 is the most common $(31.00 \%+11.25 \%=42.25 \%)$, but because it is unlikely that two modules will be needed at once (see Table 2), the best strategy is to first attempt to assign a unique case to each module.

Whenever the number of instructions of a particular case exceeds the number of modules reserved for that case, then it is necessary to place some of them in non-ideal modules. These overflow situations are dealt with in the order of their probability of occurring. The strategy for making non-ideal assignments is to greedily choose the module that is likely to incur the smallest cost. 


\subsection{Operand Swapping}

We also propose a method for swapping operands without considering the previous inputs; the intuition of our method is as follows. The most power-consuming integer computations are those where the information bits for both operands fail to match the bits from the previous operands to that FU. There are four ways that this may occur: case 00 follows case 11 , case 11 follows case 00 , case 10 follows case 01 , or case 01 follows case 10 . In the last two of these, swapping the operands converts a worst-case situation into a best-case situation, assuming that the operation is commutative.

Therefore, we propose always swapping the operands for one of these cases. To minimize mismatches, the case to swap from should be the one that has the lower frequency of non-commutative instructions. Only non-commutative instructions are considered, because these are the ones that will not be flipped, and will therefore cause mismatches. Table 1 shows that for the IALU, the $4_{t h}$ row has a lower frequency than the $6_{t h}$; for the FPAU, the $6_{t h}$ row is the smaller. Therefore, case 01 instructions will be swapped for the IALU, and case 10 instructions for the FPAU.

Compiler-based swapping An alternative strategy for swapping operands is to perform it in software, by physically changing the machine instructions in the binary executable, using profiling. This approach is not particularly novel, but is studied so as to avoid the hardware cost of dynamic operand swapping. It is also instructional, in that our results show that the benefit of our hardware method is fairly independent of this transformation.

Compiler-based swapping has three advantages over hardware swapping. First, it avoids the overhead of hardware swapping. In fact, it offers some power improvement even when our hardware mechanism is not implemented. Second, the compiler can afford to count the full number of high bits in the operands, rather than approximating them by single bit values. For example, a 
" $1+511$ " operation and a " $511+1$ " operation both look like a case 00 to our hardware method. A compile-time method, however, can recognize the difference and swap when beneficial. Third, certain operations are commutable by the compiler but not by the hardware. An example is the ">" operation, which can become the $\leq$ operation when the operands are swapped. The compiler is able to change the opcode, but the current hardware strategy cannot.

But the compiler approach also has three main disadvantages. First, each instruction's operands are either always swapped or always not. The decision is made based on the average number of high bits for the two operands. In contrast, the hardware technique captures the dynamic behavior of the instruction. Hardware can choose to swap an instruction's operands on one cycle, and not to swap them on a later cycle. Second, since the program must be profiled, performance will vary somewhat for different input patterns. Third, some instructions are not commutative in software. One example is the immediate add. While this operation is commutative, there is no way to specify its operand ordering in the machine language - the immediate value is always taken to be the second.

Since both hardware and compiler swapping have their advantages, the best results are achieved with both.

Swapping for multiplier units Since even modern superscalars tend to only have one integer and one floating point multiplier, reducing power by steering to one of multiple functional units is not feasible for multiplication. However the following observation allows for savings using a different approach: in the case of the Booth multiplier, the power is known to depend not only on the switching activity of the operands, but also on the number of 1's in the second operand [15]. The underlying reason for this observation is that the Booth multiplier is an optimized version of the elementary shift-and-add multiply algorithm taught in introductory digital logic courses, which 
needs to add only when the current bit in the second operand is one. We observe that power savings are possible by swapping the operands to ensure that the second operand is the one with fewer ones in it. Swapping can be done either by hardware or by the compiler, with the corresponding advantages and disadvantages listed earlier in this section.

Unfortunately we do not have a simple high-level power model for the Booth multiplier, and thus we do not measure the power improvements from swapping for multiplication. Nevertheless table 3 shows the potential for improvement by listing the bit patterns for multiplication data. This table shows that $15.5 \%$ of floating point multiplications can be swapped from case 01 to case 10 , certainly resulting in some additional power savings - though not quantifiable since we have no power model for multiplication.

\section{Practical considerations}

We now consider the costs of our method. The potential costs are (1) increased power and area due to additional buses and logic, and (2) increased cycle time due to more complex routing logic. In fact, additional buses are not needed, and the increase in logic is not large.

In appraising the costs of the method, it is helpful to review how FUs are currently assigned by a superscalar processor. The most common methods are loosely based upon Tomasulo's algorithm [11], where each FU type has its own reservation station. On each cycle, those instructions whose operands become ready are removed from the reservation station and assigned to the FUs on a first-come-first-serve basis. Figure 3 shows the basic hardware used in Tomasulo's algorithm. This algorithm allows instructions to execute out of order, so it requires routing logic and a crossbar. Since, on existing machines, most stations must already be able to map to any module, we do not need to add new bus wires. The information bits, however, must be sent to the routing 
logic. Although the number of wires needed for this is small, their cost can be reduced by only forwarding information bits from some of the reservation stations. For example, if there are many reservation station entries, then it is unlikely that the higher ones will be filled. For a 4-bit LUT, only enough wires are needed to make it probable that the routing logic receives information bits for the first two operations issued.

It is unavoidable, however, that our method increases the complexity of the routing control logic. We replace the existing simple routing logic with the not-as-simple LUT described above. To make the LUT smaller and faster, we propose reducing the size of the vector. Since Num(I) $<$ $\operatorname{Num}(\mathrm{M})$ for most cycles, it is reasonable to consider a smaller vector that may not always contain all of the instructions. Reducing the size of the vector makes the proposed hardware modification faster and therefore more attractive. In the results we show that a 4-bit vector yields good performance. With a 4-bit vector, our fully implemented algorithm for the IALU, on a machine with 8 entries in its reservation station, requires 58 small logic gates and 6 logic levels. With 32 entries, 130 gates and 8 levels are needed. This is a negligible fraction of the many thousands of gates present in the IALU. Therefore, the power and delay introduced are small.

A third issue is that the crossbar currently used in Tomasulo's algorithm does not allow operand swapping. To perform operand swapping, it would be necessary to include additional wiring after the crossbar of Figure 3. This makes the compiler-based swapping methods more attractive.

\section{Experimental Results}

We have implemented the methodology described in section 4 using the sim-outorder simulator from the SimpleScalar 2.0 suite [5], with the default configuration of 4 IALUs, 4 FPAUs, 1 integer multiplier, and 1 floating point multiplier. SimpleScalar simulates a MIPS-like machine, 
with 32-bit integer registers and 64-bit floating point registers. The integer benchmarks used are: 88ksim, ijpeg, li, go, compress, cc1, and perl. The floating point benchmarks are: apsi, applu, hydro2d, wave5, swim, mgrid, turb3d, and fpppp. The benchmarks are from SPEC 95 [9], and were run to completion on large input files.

Figure 4 displays the power reduction of different schemes, as a fraction of the total power consumption of the FU type under consideration. Figure 4(a) shows the numbers for the integer ALU and figure 4(b) shows the numbers for the floating point arithmetic unit. Each bar is a stack of three values, so as to show the effect of operand swapping on the switching activity (which loosely approximates energy). Full Ham (section 4.1) and 1-bit Ham (section 4.2) are cost-prohibitive, but are included for comparison. Full Ham identifies the maximum possible improvement. 1-bit Ham is an upper bound on the improvement possible solely through the information bits. The 8 bit LUT represents the approach of section 4.3. The 4-bit and 2-bit LUTs represent shortened vectors, as considered in section 5. The Original column represents a first-come-first-serve assignment strategy. The gain for Original is not zero since swapping benefits it as well.

Figure 4 provides five insights, discussed in the next five paragraphs. First, a 4-bit LUT is recommended, because its power savings are comparable to the upper bound (1-bit Ham), while being simple to implement. From Figure 4, the improvement for the 4-bit LUT with hardware swapping is $18 \%$ for the FPAU and $17 \%$ for the IALU. With compiler swapping it is $26 \%$ for the IALU. If no swapping of any kind is used, the still-rather-simple 8-bit LUT yields a similar result to the 4-bit LUT with hardware swapping. In all cases, we see a significant improvement in power consumption by using a 4-bit LUT versus the original configuration.

Second, figure 4(b) shows that the FPAU does not benefit much from operand swapping. In all cases, almost all the improvement can be gained using the base method of operand steering to 
particular FUs, without swapping. The reason is that, for integers, the majority of bits are usually the same as the information bit; but for floating points, only an information bit value of zero has this property. This is because, in floating point, if the OR of the bottom four bits equals zero, then many trailing bits are expected to be zero, but the opposite is not true. In particular, if the $O R$ result is one, then the floating point value is likely a full-precision value and no predominant value can be predicted in the trailing bits; they are likely 50-50\% split between zeros and ones. Thus, for the IALU, a case 01 after a 10 causes most bits to switch; where as a case 01 followed by a 01 (resulting from a swapping of the 10 case) switches few bits, showing the benefit from swapping. In contrast, for the FPAU, a 01 case after a 10 switches $\frac{1}{2}$ of the bits; where as a case 01 followed by a 01 (resulting from a swapping of the 10 case) still switches $\frac{1}{4}$ of the bits. Thus there is only a $\frac{1}{4}$ fraction improvement from swapping, which explains its low benefit for floating point.

Third, for integers, profile-based swapping is more effective that hardware-based swapping, because the swap decision is based on the entire value, as opposed to the information bits alone. In fact, "Base + Compiler Swapping" (not shown) is nearly as effective as "Base + Hardware + Compiler". For floating point, all kinds of swapping are ineffective, as discussed above.

Fourth, for integers, implementing compiler swapping does not reduces the additional benefit of our hardware approach. Rather, the benefit of compiler swapping is slightly higher with an 8-bit LUT than it is for the original processor that has no hardware modifications.

Fifth, the FPAU is less sensitive to the size of the LUT access vector (4 or 8), because the floating point unit is less heavily loaded per cycle (Table 2). Only when three or more floating point instructions execute in a cycle, which is rare for floating point, is there a benefit from an 8-bit LUT over a 4-bit LUT.

We do not measure the power savings from our proposed optimization for floating point and 
integer multiplier units. This is because we do not have a good power model for the Booth multiplier we optimize for, and thus the power benefits are difficult to quantify. Numbers quantifying the potential cases for improvement were presented in section 4.4.

\section{Conclusions}

This paper presents some architectural-level schemes for reducing dynamic power in superscalar processors. We present a method for assigning operations to functional units, so as to reduce power consumption for those units with duplicated modules. We also examined operand swapping, both in conjunction with our assignment algorithm, and on its own merits for non-duplicated functional units like the multiplier. The basic intuition of most of our methods is to reduce the number of bits whose values change between successive computations on the same functional unit.

Toward this end, we make define a low-cost way of predicting which bit value, zero or one, is prevalent in a single integer or floating-point value without actually examining all the bits. For integer values the sign bit is a good predictor because of sign-extension in twos complement. For floating point values the OR of the lowest four bits of the mantissa is a good predictor. The reasons for these are elaborated in sections 1 and 4 . The bit used to predict the frequency of the rest is called the information bit.

Based on these observations we make the following four contributions. First, we present a method for integer ALUs that steers operations to one of multiple integer ALUs, each specialized for certain information bit combinations. Since operations with the same information bit combination are mapped to the same ALU, and their operands have a similar frequency of zeros and ones, the number of bit value changes is lowered significantly, thereby reducing power. Second, we present a method for floating point adders and subtractors that is identical to that for integers, 
but with a different definition of information bit (defined above). Third, we present how operand swapping benefits integer ALU operations, both using compiler and hardware swapping. Swapping is not found to effective for floating point, and we present a reason why in the results section. Fourth, we present a method for reducing power in integer and floating point multipliers by using swapping (section 4.4), but lacking a power model, no power reduction results are presented.

Our results show that these approaches reduce $17 \%$ of the IALU and $18 \%$ of the FPAU switching, with only a small hardware change. Compiler swapping increases the IALU gain to $26 \%$.

\section{References}

[1] M. Alidina, J. Monteiro, S. Devadas, and M. Papaefthymiou. Precomputation-Based Sequential Logic Optimization for Low Power. IEEE Transactions on VLSI Systems, 2(4):426-436, April 1994.

[2] L. Benini and G. D. Micheli. Transformation and Synthesis of FSMs for Low Power Gated Clock Implementation. IEEE Transactions on Computer-Aided Design, 15(6):630-643, June 1996.

[3] D. Brooks and M. Martonosi. Dynamically Exploiting Narrow Width Operands to Improve Processor Power and Performance. In Proc of the 5th Int'l Symp on High Performance Computer Architecture (HPCA), pages 13-22, January 1999.

[4] D. Brooks, V. Tiwari, and M. Martonosi. Wattch: A Framework for Architectural-Level Power Analysis and Optimizations. In Proceedings of the 27th International Symposium on Computer Architecture (ISCA), Vancouver, British Columbia, June 2000.

[5] D. Burger and T. Austin. The SimpleScalar Tool Set, Version 2.0. Technical Report TR 1342, University of Wisconsin, Madison, WI, June 1997.

[6] J.-M. Chang and M. Pedram. Module Assignment for Low Power. In Proc of the European Conference on Design Automation (EDAC), pages 376-381, September 1996.

[7] O.-C. Chen, R.-B. Sheen, and S. Wang. A low-power adder operating on effective dynamic data ranges. IEEE Transactions on Very Large Scale Integration (VLSI) Systems, 10(4):435-453, August 2002. 
[8] J. Choi, J. Jeon, and K. Choi. Power minimization of functional units partially guarded computation. In Proceedings of the 2000 international symposium on Low power electronics and design, pages 131-136. ACM Press, 2000.

[9] S. P. E. Corporation. The SPEC benchmark suites. http://www.spec.org/.

[10] S. Dropsho, V. Kursun, D. H. Albonesi, S. Dwarkadas, and E. G. Friedman. Managing static leakage energy in microprocessor functional units. In Proceedings of the 35th annual ACM/IEEE international symposium on Microarchitecture, pages 321-332. IEEE Computer Society Press, 2002.

[11] J. Hennessy and D. Patterson. Computer Architecture A Quantitative Approach. Morgan Kaufmann, Palo Alto, CA, third edition, 2002.

[12] M. Johnson, D. Somasekhar, and K. Roy. Leakage Control with Efficient Use of Transistor Stacks in Single Threshold CMOS. In Design Automation Conference, pages 442-445, June 1999.

[13] B. Klass, D. E. Thomas, H. Schmit, and D. E. Nagle. Modeling Inter-Instruction Energy Effects in a Digital Signal Processor. In Power-Driven Microarchitecture Workshop, in conjunction with Int'l Symposium on Computer Architecture, June 1998.

[14] L. Kruse, E. Schmidt, G. Jochenshar, and W. Nebel. Lower and Upper Bounds on the Switching Avtivity in Scheduling Data Flow Graphs. In Proc of the ACM Int'l Symp on Low Power Design, pages 115-120, August 1999.

[15] T.-C. Lee, V. Tiwari, S. Malik, and M.Fujita. Power Analysis and Minimization Techniques for Embedded DSP Software. IEEE Transactions on VLSI Systems, Mar. 1997.

[16] R. Marculescu, D. Marculescu, and M. Pedram. Sequence compaction for power estimation: Theory and practice. IEEE Transactions on Computer Aided Design, 18(7):973-993, 1999.

[17] J. Mermet and W. Nebel. Low Power Design in Deep Submicron Electronics. Kluwer Academic Publishers, Norwell, MA, 1997.

[18] M. Pedram. Power Minimization in IC Design: Principles and Applications. ACM Transactions on Design Automation of Electronic Systems, 1(1):1-54, January 1996.

[19] J. S. Seng, E. S. Tune, and D. M. Tullsen. Reducing power with dynamic critical path information. In Proceedings of the 34th annual ACM/IEEE international symposium on Microarchitecture, pages 114-123. IEEE Computer Society, 2001. 
[20] D. Shin, J. Kim, and S. Lee. An Operational Rearrangement Technique for Power Optimization in VLIW Instruction Fetch. In Proceedings of Design, Automation \& Test in Europe (DATE 2001), March 2001.

[21] V. Tiwari, S. Malik, and P. Ashar. Guarded Evaluation: Pushing Power Management to Logic Synthesis/Design. In Proceedings of the ACM/IEEE International Symposium on Low Power Design, pages 139-142, April 1994. 
Table 1: Bit patterns in data The values in the first three columns are used to separate the results into 8 rows. Columns 1 and 2 show the information bits of both operands; (for integers, the top bit; for floating points, the OR-ing of the bottom four bits of the mantissa). Columns 4 and 7 are the occurrence frequencies for the given operand bits and commutativity pattern, as a percentage of all executions of the FU type. Columns 5, 6, 8, and 9 display, for the specified operand, the probability of any single bit being high.

\begin{tabular}{|c|c|c||c|c|c||c|c|c|}
\hline $\mathrm{O}$ & $\mathrm{O}$ & Com- & \multicolumn{3}{|c||}{ IALU } & \multicolumn{3}{c|}{ FPAU } \\
\cline { 2 - 8 } $\mathrm{P}$ & $\mathrm{P}$ & muta- & Freq & OP1 & OP2 & Freq & OP1 & OP2 \\
1 & 2 & tive & $(\%)$ & prob & prob & $(\%)$ & prob & prob \\
\hline 0 & 0 & Yes & 40.11 & .123 & .068 & 16.79 & .099 & .094 \\
0 & 0 & No & 29.38 & .078 & .040 & 10.28 & .107 & .158 \\
0 & 1 & Yes & 9.56 & .175 & .594 & 15.64 & .188 & .522 \\
0 & 1 & No & 0.58 & .109 & .820 & 4.90 & .132 & .514 \\
1 & 0 & Yes & 17.07 & .608 & .089 & 5.92 & .513 & .190 \\
1 & 0 & No & 1.51 & .643 & .048 & 4.22 & .500 & .188 \\
1 & 1 & Yes & 1.52 & .703 & .822 & 31.00 & .508 & .502 \\
1 & 1 & No & 0.27 & .663 & .719 & 11.25 & .507 & .506 \\
\hline
\end{tabular}


Table 2: Frequency that the functional unit uses a particular number of modules for a 4-way machine with 4 IALUs and 4 FPAUs. There is no $\operatorname{Num}(\mathrm{I})=0$ column because we only consider cycles which use at least one module - the other case being unimportant to power consumption within a module (ignoring leakage).

\begin{tabular}{|r|c|c|c|c|}
\hline Num(I) $=$ & 1 & 2 & 3 & 4 \\
\hline IALU & $40.3 \%$ & $36.2 \%$ & $19.4 \%$ & $4.2 \%$ \\
FPAU & $90.2 \%$ & $9.2 \%$ & $0.5 \%$ & $0.1 \%$ \\
\hline
\end{tabular}


Table 3: Bit patterns in multiplication data (Multiplier power is related to how many 01 cases can become 10.)

\begin{tabular}{|c||c|c|c||c|c|c|}
\hline \multicolumn{1}{|c||}{} & \multicolumn{3}{c||}{ Integer } & \multicolumn{3}{c|}{ Floating Point } \\
\cline { 2 - 7 } Case & $\begin{array}{c}\text { Freq } \\
(\%)\end{array}$ & $\begin{array}{c}\text { OP1 } \\
\text { prob }\end{array}$ & $\begin{array}{c}\text { OP2 } \\
\text { prob }\end{array}$ & $\begin{array}{c}\text { Freq } \\
(\%)\end{array}$ & $\begin{array}{c}\text { OP1 } \\
\text { prob }\end{array}$ & $\begin{array}{c}\text { OP2 } \\
\text { prob }\end{array}$ \\
\hline 00 & 93.79 & 0.116 & 0.056 & 20.12 & 0.139 & 0.095 \\
01 & 1.07 & 0.055 & 0.956 & 15.52 & 0.160 & 0.511 \\
10 & 2.76 & 0.838 & 0.076 & 21.29 & 0.527 & 0.090 \\
11 & 2.38 & 0.71 & 0.909 & 43.07 & 0.274 & 0.271 \\
\hline
\end{tabular}




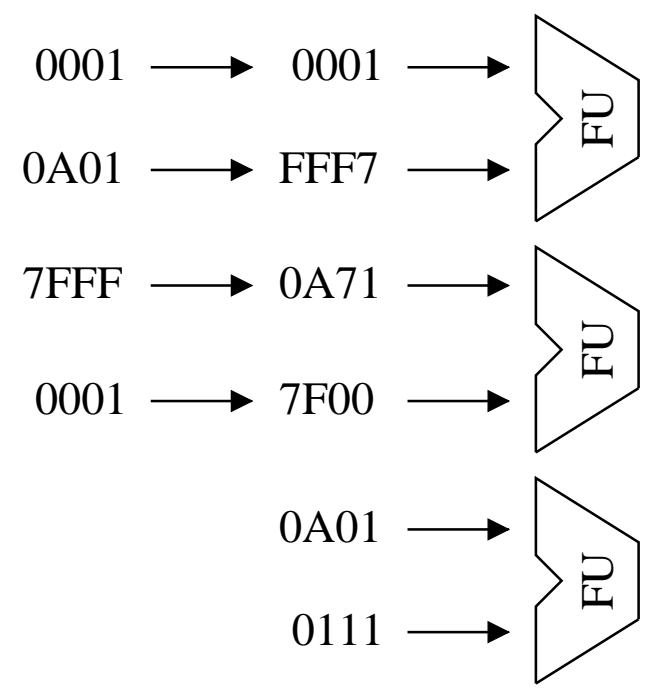

cycle $2 \quad$ cycle 1

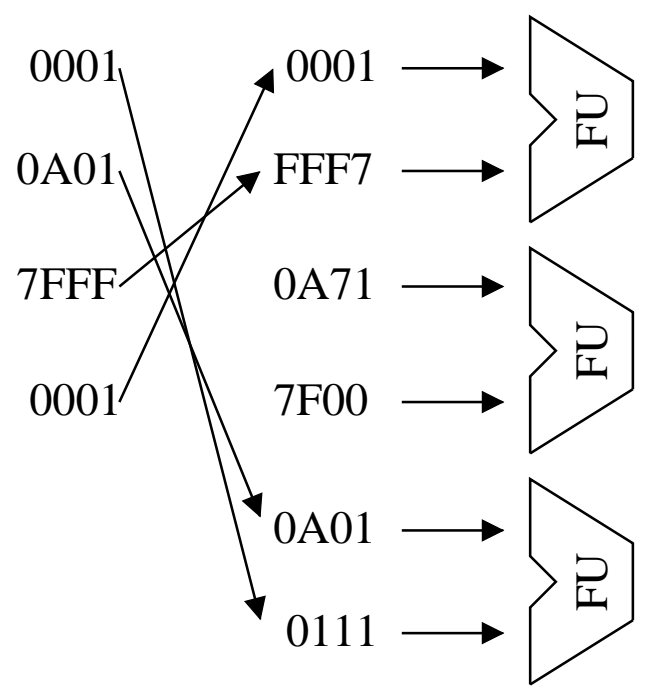

cycle $2 \quad$ cycle 1

\section{Default routing}

Alternative routing (57\% less energy)

Figure 1: Alternative data routes for a 3-way processor. 


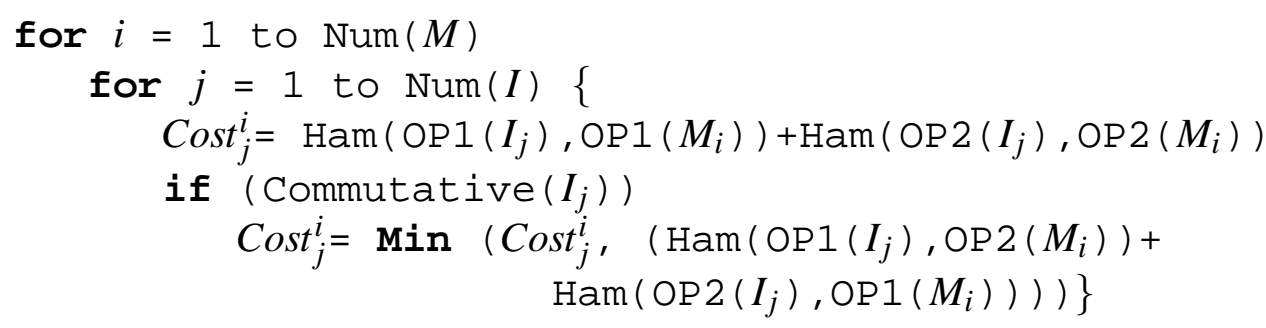

Figure 2: Finds the cost of every possible assignment. 


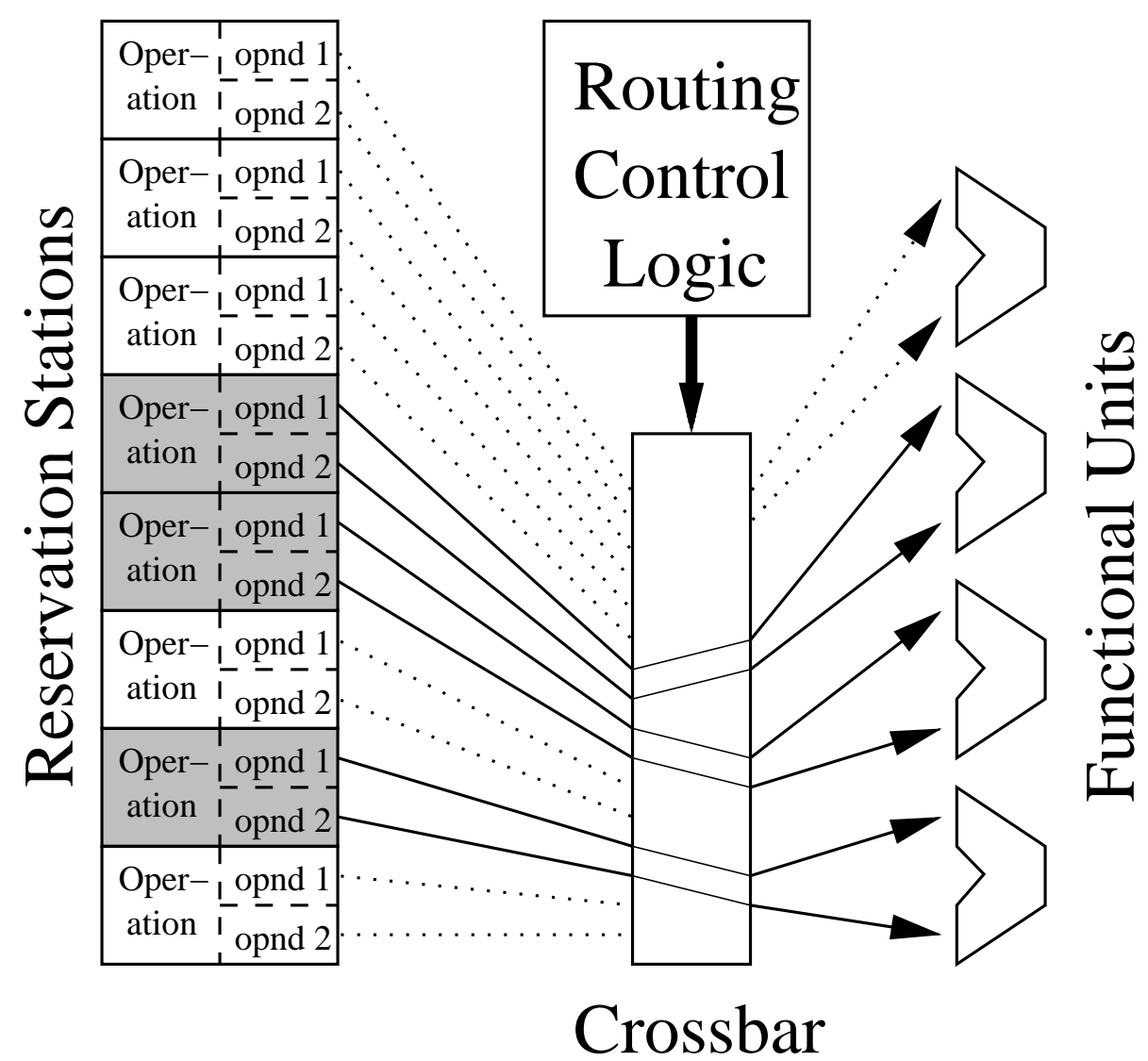

Figure 3: On a particular cycle, 3 operations are shaded to show they are ready to execute. Dotted lines indicate inactive buses, while solid lines represent active ones. Operations indicate their readiness to the routing control logic, which in turn schedules them, in order, to the FUs. The key observation is that the crossbar implies that no new buses will be needed by our method. 


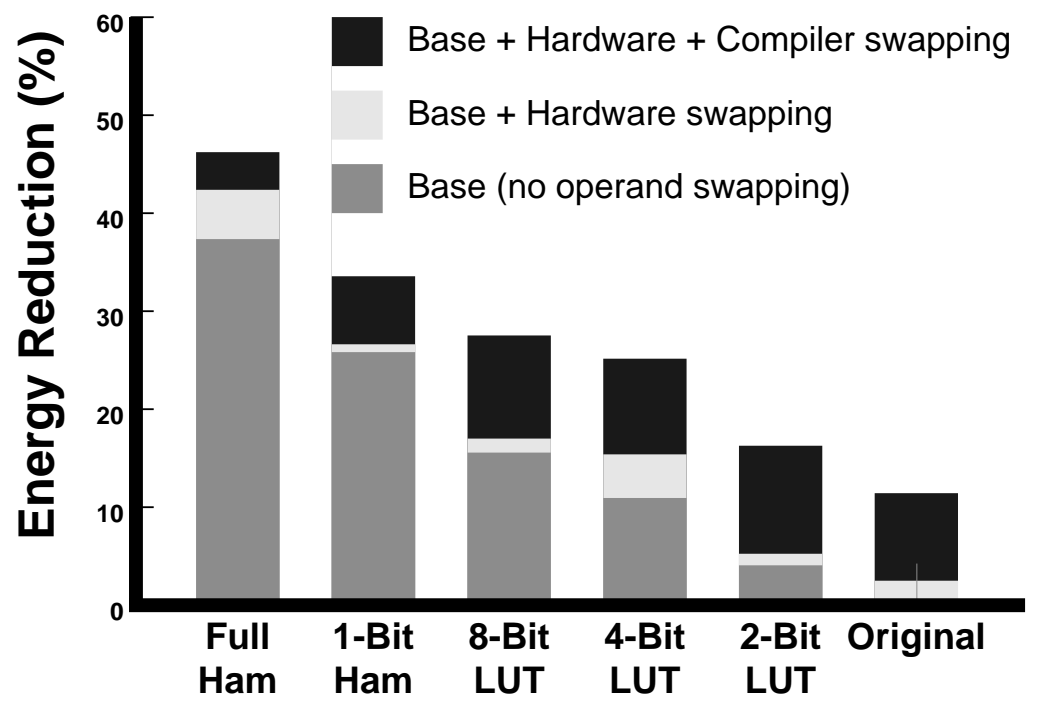

(a)

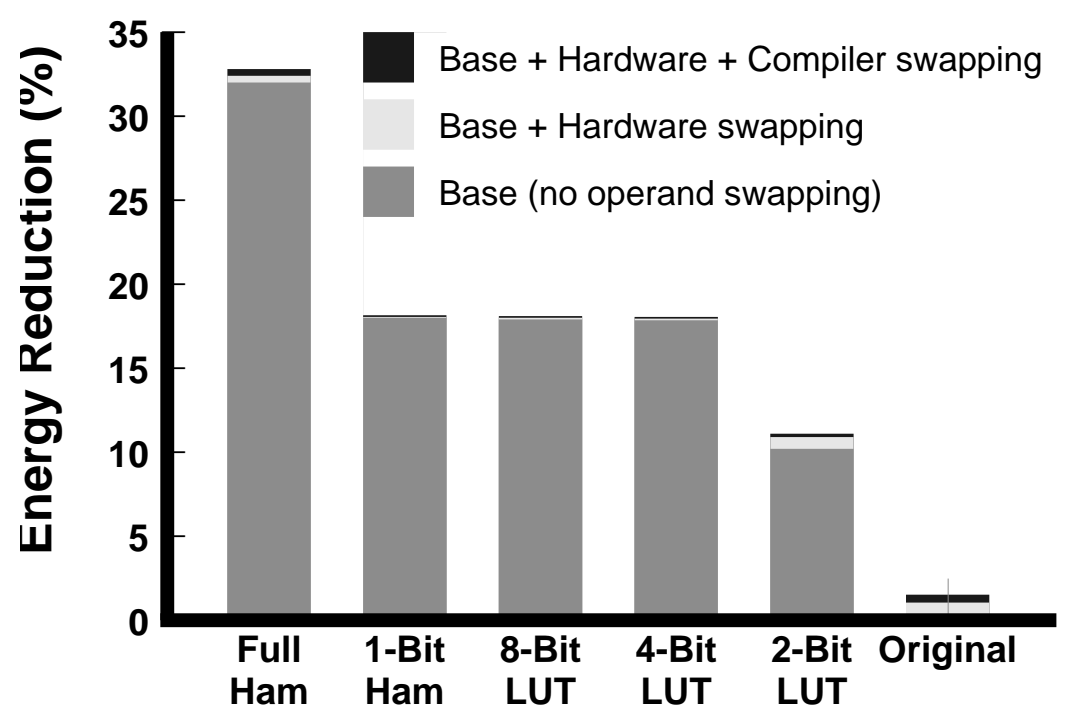

(b)

Figure 4: Results for the (a) IALU and (b) FPAU. 